\title{
How healthtech is transforming the future of rare disease diagnosis
}

\author{
Rudy Benfredj $*, 1$ \\ ${ }^{1}$ Mendelian 239 old Street, London, EC1V 9EY, UK \\ *Author for correspondence: rudy@mendelian.co
"Misconceptions about the role of artificial intelligence (AI) in healthcare should be dispelled, and reassurances offered that technology is an augmentative tool, designed to supplement and not substitute clinical expertise.”

First draft submitted: 20 January 2021; Accepted for publication: 29 January 2021; Published online: 10 February 2021

Keywords: diagnosis $\bullet$ rare disease $\bullet$ ultra-rare disease

Leveraging the potential of technology in healthcare has long been dismissed as a pipedream, held back by a reluctance to adopt change.

Despite its promise in many circles, the advent of technology in health has, at times, come under particular scrutiny, its critics perceiving it as abstract and/or ineffectual. Yet for all the expectations and rhetoric, it appears the promise of digital health is finally being realized. There has been a palpable narrative shift from "what can technology do for healthcare in the future?" to "what is technology doing for healthcare today?".

Healthtech is at an inflection point, due in no small part to the convergence between the ambitions of clinicians, researchers and the commercial sector, with a degree of central oversight and commitment never before seen. The emphasis on digital technologies in the National Health Service (NHS) in the form of NHS Long Term Plan, together with the establishment of NHSX and its various composite arms (such as the NHS AI Lab), evidences an unprecedented desire from the top to harness the promise of digital in healthcare [1]. In the rare disease field, the recently published Genome UK report has served to embolden the various actors in this space [2]. The report offers the assurance that rare disease diagnosis and management is not in the esoteric interests of a few, but rather a national health policy priority.

The deployment of digital health solutions in the rare disease field does, nonetheless, pose challenges of capacity building, which must be effectively navigated if the technology is to be embedded within routine care. This commentary outlines Mendelian's efforts to improve rare disease diagnosis, presents a vision for the future impact and scope of digital health in the context of rare diseases, and considers technical and cultural factors that must concurrently be addressed if maximal value from digital is to be derived.

\section{The challenge of rare disease diagnosis}

'Rare disease' has, regrettably, been historically low on the public health agenda, a fact not lost on the 350 million people suffering with these diseases globally [3]. This is unsurprising; individually, each of these rare diseases exert too small a health burden to warrant an easy health-economic case, yet as a collective, rare diseases are understood to be as prevalent as common respiratory conditions such as asthma, with over 8000 rare diseases affecting one in 17 people worldwide [3].

The dearth of expertise and knowledge in the rare diseases field has meant that patients often wait several years for a diagnosis (if at all), frequently receiving misdiagnoses and undergoing unnecessary, invasive investigations. Clinicians are all too familiar with the case of the 'pyrexia of unknown origin' patient [4]; investigated head-to-toe with all number of biochemical assays and imaging modalities drawing a blank, only to be discharged home with neither doctor nor patient any the wiser. This is occurring far too often, and at too great a cost, to continue to be overlooked. 
At a broader health system level, the economic costs of rare diseases are significant. It is estimated that undiagnosed rare diseases have cost the NHS over $£ 3.4$ billion in the past decade, with $10 \%$ of the NHS budget in 2016 spent on rare diseases [5,6]. Patients with rare diseases are understood to cost a hospital $£ 13,000$ each over 10 years, almost double the costs associated with other, more prevalent, diseases [5].

\section{About Mendelian}

Mendelian was founded in 2015 by a team composed of a neurologist, computer scientists and seasoned entrepreneurs with the aim of supporting clinicians to identify rare diseases sooner. Recognizing the health and economic consequences of delayed diagnosis and time to intervention, the team developed the MendelScan solution, which uses state-of-the-art technologies in data capture and the latest medical knowledge to enable and improve rare disease diagnosis. The tool extracts signs and symptoms from electronic health records (EHRs) across a patient population, and is the first NHS-integrated tool built especially to support primary care diagnosis of rare disease. The algorithms are based on national guidelines, and have been developed in conjunction with ten international experts in rare disease and 19 patient support groups. To date, diagnostic criteria for over 100 rare diseases have been encoded.

Patients flagged as potentially having a rare diagnosis are highlighted and an extended medical history review is performed by Mendelian's clinical team and disease specialists. The information is used to generate a MendelScan clinical report describing the suspected disease and appropriate NHS diagnostic pathway. Healthcare providers are then encouraged to invite the patient for review and combine their clinical expertise with the novel insights from the report to determine the appropriate course of action.

In a series of retrospective case studies, Mendelian demonstrated the potential cost and time savings in rare disease diagnosis had the MendelScan solution been deployed. For example, for a patient with Behcet's disease - a rare vasculitic disease manifesting most commonly with orogenital ulceration - the MendelScan could have brought forward the time to diagnosis by 4 years, avoided three unnecessary referrals and one misdiagnosis, and reduced the cost of the diagnostic odyssey by $76 \%$ [7]. Similarly for a patient with the connective-tissue disorder Ehlers-Danlos syndrome, MendelScan offered the potential to make the diagnosis 3 years earlier, avoid four unnecessary referrals and two general practitioner (GP) visits, and reduce the cost of diagnosis by $35 \%$ (internal study; Unpublished Data) [8].

The recently announced partnership between Mendelian and Modality NHS Partnership reflects the next step in Mendelian's efforts to deploy their solution at scale. Modality is the largest GP super partnership in the United Kingdom comprising 450,000 patients from 45 practices across the country. As the coverage and capability of the MendelScan algorithm grows, including integration within secondary care systems, an ever-increasing number of patients can stand to benefit from timely diagnosis and earlier time to treatment.

\section{Genome UK report}

The scalability and sustainability of actors such as Mendelian is contingent on central oversight and a commitment from the top. The recently published Genome UK report was a source of great encouragement, in many ways a call-to-action for stakeholders interested in challenging the rare disease status quo [2]. From the advent of the 100,000 genomes project by Genomics England in 2012, to the $£ 200$ million Whole Genome Sequencing project which aims to sequence the genetic code of 500,000 UK Biobank volunteers, the United Kingdom has consistently been a trailblazer in genomic medicine, with other nations quick to follow.

The Genome UK report outlines three pillars underpinning its vision and strategy for the future: diagnosis and personalized medicine, prevention, and research. The report further identifies rare and inherited diseases as a key priority, alongside cancer, pharmacogenomics and infectious diseases. With over $80 \%$ of rare diseases having a genetic component, a renewed commitment to genomic medicine is cause for excitement [9]. The report sets out several key aims in the rare disease field, including improving the diagnostic rate from genomic testing, expediting the time to diagnosis, greater provision of Whole Genome Sequencing services and increasing the number of patients with rare diseases enrolled on specialist clinical trials.

It is important to recognize that the relationship between genomic research and clinical medicine is bidirectional and mutually reinforcing: phenotypic variants suggestive of an underlying disease process should be presented for genomic testing, and likewise genomic variations may help to explain phenotypic features. To that end, collaboration between researchers and clinicians within the broader agenda of nurturing a learning environment is centrally important in raising awareness and promoting the growth of genomic medicine. 


\section{Vision for the future}

The ultimate aim of publications such as the Genome UK report, initiatives such as the $100 \mathrm{~K}$ Genome project, accelerators such as Illumina and health-tech startups such as Mendelian, is to translate research from bench-tobedside and to embed our understanding of this nascent science within routine clinical practice.

Routinizing the rare disease field serves to improve access, as healthcare becomes more equitable and expertise democratized. Indeed the interplay between policy, research and innovation largely becomes a question of ecosystem building, reflecting a concerted effort to transition from rare diseases as the clandestine remit of a few expert clinicians, toward becoming everybody's business. The epidemiological prevalence of a disease should have no bearing on the expertise and knowledge base that exists around it, with clinicians in the future managing rare diseases as proficiently as they do asthma or hypertension.

The absorptive capacity to embed digital health solutions to rare diagnosis is particularly dependent on two key rate-limiting steps. The first is concerned with having a serviceable in situ technical infrastructure to derive the most benefit from healthcare data. If digital algorithms are the engine pushing forward the health informatics agenda, a robust EHR is the chassis atop which they must sit. Increased investment in standardized, streamlined EHRs with improved interoperability will facilitate the data extraction and analysis necessary for solutions such as MendelScan to deliver the most value for patients and health systems.

The second, arguably more important bottleneck, is a question of culture. It is essential to increase awareness among healthcare professionals of the adjunctive and assistive role of data-driven technologies to support their clinical practice. Misconceptions about the role of artificial intelligence (AI) in healthcare should be dispelled, and reassurances offered that technology is an augmentative tool, designed to supplement and not substitute clinical expertise. Similarly, patients should be informed of the value that exists in their healthcare data, the diagnostic clues hiding in plain sight, waiting to be joined up (whether through technology or otherwise) for the benefit of their health and wellbeing.

Notwithstanding the significant gains made in our understanding of personalized medicine, genomic research continues to be a somewhat esoteric field of study, inaccessible to the average clinician. As such, a greater understanding of this area will serve to empower our frontline doctors, as they grow cognizant of the patients whom to refer for genomic testing, and the therapies to offer based on the results of gene sequencing. This will require increased continuing professional development and postgraduate training opportunities made available to healthcare professionals.

\section{Conclusion}

Digital health solutions have improved exponentially in recent years, and it no longer seems premature to conclude that they have transitioned from the abstract to the achievable. Rare disease diagnosis and management is one such field that has benefited as a result of the great strides made in technology, as exemplified by Mendelian and the MendelScan algorithms. Increasing government commitment toward moving digital further up the health policy agenda has served to reassure innovators and investors that their interests are protected. Similarly, exciting developments continue to emerge in the genomic medicine area, which augur well for our understanding of the pathophysiology and pharmacogenomics of rare diseases. As we strive toward integrating these fields of study within routine clinical practice, concurrent investment in upgrading our technology infrastructure and facilitating the education of our workforce is of equal importance.

Financial \& competing interests disclosure

R Benfredj is the CEO of Mendelian. The author has no other relevant affiliation or financial involvement with any organization or entity with a financial interest in or financial conflict with the subject matter or materials discussed in the manuscript apart from those disclosed.

Medical writing support was provided by B Chada and was funded by SomX (communications agency for Mendelian).

\section{Open access}

This work is licensed under the Attribution-NonCommercial-NoDerivatives 4.0 Unported License. To view a copy of this license, visit http://creativecommons.org/licenses/by-nc-nd/4.0/ 


\section{References}

1. NHS. The NHS long term plan. (2019). www.longtermplan.nhs.uk/wp-content/uploads/2019/08/nhs-long-term-plan-version-1.2.pdf

2. HM Government. GENOME UK: the future of healthcare. (2020). https://assets.publishing.service.gov.uk/government/uploads/system /uploads/attachment_data/file/920378/Genome_UK_-_the_future_of_healthcare.pdf

3. Project8p.org. Rare disease facts. https://project8p.org/rare-disease-facts/

4. Fernandez C, Nick JB. Pyrexia of unknown origin. Clin. Med. 18(2), 170-174 (2018).

5. Harrison K. Imperial College Health Partners. New report reveals that, while undiagnosed, rare disease patients have cost the NHS in excess of $£ 3.4$ billion: Imperial College Health Partners. (2018).

https://imperialcollegehealthpartners.com/new-report-reveals-undiagnosed-rare-disease-patients-cost-nhs-excess-3-4-billion/

6. DigitalHealth.London. Mendelian - DigitalHealth.London. https://digitalhealth.london/accelerator/companies/2020-21/mendelian/

7. Buendia O, Benfredj R, Halford T et al. Case report: reducing the diagnostic odyssey in Behcet's disease using a digital health approach into primary care UK. www.mendelian.co/pdf/poster-reducing-the-diagnostic-odyssey.pdf

8. Mendelian.co. Our solution for rare diseases | Mendelian.co. www.mendelian.co/solution

9. Genomics England. Rare disease genomics | Genomics England. www.genomicsengland.co.uk/understanding-genomics/rare-disease-genomics/ 